\title{
LncRNA FEZF1-AS1 promotes colorectal cancer progression through regulating the miR-363-3p/PRRX1 pathway
}

\author{
Tongtong Zhang ${ }^{\mathrm{A}, \mathrm{D}}$, Suyang $\mathrm{Yu}^{\mathrm{B}, \mathrm{C}}$, Shipeng Zhao ${ }^{\mathrm{E}, \mathrm{F}}$ \\ Department of Gastrointestinal Surgery, The Third Hospital Affiliated to Hebei Medical University, Shijiazhuang, China \\ A - research concept and design; $\mathrm{B}$ - collection and/or assembly of data; $\mathrm{C}$ - data analysis and interpretation; \\ $D$ - writing the article; $E$ - critical revision of the article; $F$ - final approval of the article
}

Address for correspondence

Shipeng Zhao

E-mail:df452355@126.com

Funding sources

None declared

Conflict of interest

None declared

Received on February 6, 2021

Reviewed on March 3, 2021

Accepted on April 10, 2021

Published online on July 20, 2021
Cite as

Zhang T, Yu S, Zhao S. LncRNA FEZF1-AS1 promotes colorectal cancer progression through regulating the miR-363-3p/PRRX1 pathway. Adv Clin Exp Med. 2021;30(8):839-848.

doi:10.17219/acem/135693

DOI

10.17219/acem/135693

\section{Copyright}

Copyright by Author(s)

This is an article distributed under the terms of the

Creative Commons Attribution 3.0 Unported (CC BY 3.0)

(https://creativecommons.org/licenses/by/3.0/)

\begin{abstract}
Background. Long non-coding RNAs (IncRNAs) are involved in the development of many cancers, including colorectal cancer (CRC). FEZ family zinc finger 1 antisense RNA 1 (FEZF1-AS1) is a key IncRNA in the regulation of CRC progression, but its potential molecular mechanisms need to be further explored.

Objectives. To investigate the mechanism of IncRNA FEZF1-AS1 in the progression of CRC.

Materials and methods. Quantitative real-time polymerase chain reaction (qRT-PCR) was performed to measure FEZF1-AS1 and miR-363-3p expression. Cell proliferation, migration and invasion were analyzed using Cell Counting Kit-8 (CCK-8) and transwell assays. Protein expression of epithelial-mesenchymal transformation (EMT)-related markers and paired-related homeobox 1 (PRRX1) were determined using western blot analysis. The interactions among FEZF1-AS1, miR-363-3p and PRRX1 were verified with dualluciferase reporter assay. A xenograft model was constructed in vivo to confirm the role of FEZF1-AS1 in CRC tumor growth.
\end{abstract}

Results. We demonstrated that FEZF1-AS1 expression was upregulated in CRC, and its silencing reduced CRC cell proliferation, migration, invasion, and EMT. MiR-363-3p could be inhibited by FEZF1-AS1, which inhibitor could reverse the suppressive effect of FEZF1-AS1 silencing on CRC progression. Paired-related homeobox 1 could be targeted by miR-363-3p, and the inhibitory effect of FEZF1-AS1 knockdown on CRC progression could also be eliminated by PRRX1 overexpression. Furthermore, interference of FEZF1-AS1 reduced the tumor growth of $C R C$ in vivo.

Conclusions. Our data demonstrate that FEZF1-AS1 regulated PRRX1 expression to promote CRC progression via inhibition of miR-363-3p.

Key words: colorectal cancer, FEZF1-AS1, miR-363-3p, PRRX1 


\section{Background}

Globally, colorectal cancer (CRC) is one of the most common malignant tumors, with more than 1.2 million new CRC patients diagnosed annually. ${ }^{1,2}$ The age of CRC patient tends to be older (41-65 years), and is more common in females. ${ }^{3}$ At present, the leading cause of death in CRC patients was tumor metastasis, ${ }^{4}$ a process driven by epithelial-mesenchymal transformation (EMT). ${ }^{5}$ Therefore, it is urgent to explore the mechanism affecting CRC metastasis.

Currently, increasing evidence reveals that long non-coding RNAs (lncRNAs) are involved in the regulation of cancer progression, including CRC.6,7 The lncRNA is a kind of non-protein coding RNA with a length of more than 200 nucleotides (nts), ${ }^{8}$ and mainly participates in the regulation of gene expression as a competitive endogenous RNA (ceRNA) that adsorbs microRNAs (miRNAs). ${ }^{9}, 10$ Studies have shown that a variety of lncRNAs (such as NKILA, BLACAT2 and MEG3) are abnormally expressed in CRC, which can modulate its occurrence and development. ${ }^{11-13}$

FEZ family zinc finger 1 antisense RNA 1 (FEZF1-AS1) was found to be aberrantly expressed in various cancers, such as prostate cancer, retinoblastoma and oral squamous cell carcinoma. ${ }^{14-16}$ In nasopharyngeal carcinoma cells, FEZF1-AS1 silencing was discovered to significantly inhibit the EMT process of cancer cells, and played a role in promoting cancer cell proliferation and metastasis. ${ }^{17}$ Furthermore, FEZF1-AS1 has been confirmed to upregulate the invasion and EMT of human hepatocellular carcinoma cells. ${ }^{18}$ However, few studies have examined the role and potential molecular mechanism of lncRNA FEZF1-AS1 in CRC.

\section{Objectives}

Our research aimed to explore the function and mechanism of FEZF1-AS1 in CRC, so as to provide new ideas for the pathogenesis and therapy of CRC.

\section{Materials and methods}

\section{Patients and tissues}

Seventy-one CRC patients were recruited from the Third Hospital Affiliated to Hebei Medical University (Shijiazhuang, China). The clinicopathological parameters of CRC patients are shown in Table 1. The CRC tumor tissues and adjacent normal tissues were preserved at $-80^{\circ} \mathrm{C}$. Each patient provided informed consent. This study was authorized by the Ethics Committee of the Third Hospital Affiliated to Hebei Medical University and was carried out in accordance with the 1964 Declaration of Helsinki.
Table 1. Correlation between FEZF1-AS1 expression and clinicopathological parameters of colorectal cancer (CRC) patients

\begin{tabular}{|c|c|c|c|c|}
\hline Parameters & $\begin{array}{l}\text { Patients } \\
(n, \%)\end{array}$ & $\begin{array}{c}\text { FEZF1-AS1 } \\
\text { high } \\
(\mathrm{n}, \%)\end{array}$ & $\begin{array}{c}\text { FEZF1-AS1 } \\
\text { low } \\
(\mathrm{n}, \%)\end{array}$ & $\mathrm{p}$-value \\
\hline \multicolumn{5}{|c|}{ Gender } \\
\hline $\begin{array}{l}\text { Female } \\
\text { Male }\end{array}$ & $\begin{array}{l}29(40.85) \\
42(59.15)\end{array}$ & $\begin{array}{l}12(41.38) \\
24(57.14)\end{array}$ & $\begin{array}{l}17(58.62) \\
18(42.86)\end{array}$ & 0.192 \\
\hline \multicolumn{5}{|c|}{ Age [years] } \\
\hline $\begin{array}{l}\geq 60 \\
<60\end{array}$ & $\begin{array}{l}39(54.93) \\
32(45.07)\end{array}$ & $\begin{array}{l}23(58.97) \\
13(40.63)\end{array}$ & $\begin{array}{l}16(41.03) \\
19(59.37)\end{array}$ & 0.124 \\
\hline \multicolumn{5}{|c|}{ Tumor size $[\mathrm{cm}]$} \\
\hline $\begin{array}{l}\geq 4 \\
<4\end{array}$ & $\begin{array}{l}47(66.20) \\
24(33.80)\end{array}$ & $\begin{array}{c}27(57.45) \\
9(37.50)\end{array}$ & $\begin{array}{l}20(42.55) \\
15(62.50)\end{array}$ & 0.112 \\
\hline \multicolumn{5}{|c|}{ TNM stage } \\
\hline $\begin{array}{l}\text { I-II } \\
\text { III-IV }\end{array}$ & $\begin{array}{l}35(49.30) \\
36(50.70)\end{array}$ & $\begin{array}{l}13(37.14) \\
23(63.89)\end{array}$ & $\begin{array}{l}22(62.86) \\
13(36.11)\end{array}$ & $0.024^{*}$ \\
\hline \multicolumn{5}{|c|}{ Tumor grade } \\
\hline $\begin{array}{l}\mathrm{T} 1-\mathrm{T} 2 \\
\mathrm{~T} 3-\mathrm{T} 4\end{array}$ & $\begin{array}{l}30(42.25) \\
41(57.75)\end{array}$ & $\begin{array}{l}10(33.33) \\
26(63.41)\end{array}$ & $\begin{array}{l}20(66.67) \\
15(36.59)\end{array}$ & $0.012^{*}$ \\
\hline \multicolumn{5}{|c|}{ Differentiation } \\
\hline $\begin{array}{l}\text { Poor and moderate } \\
\text { Well }\end{array}$ & $\begin{array}{l}50(70.42) \\
21(29.58)\end{array}$ & $\begin{array}{l}29(58.00) \\
7(33.33)\end{array}$ & $\begin{array}{l}21(42.00) \\
14(66.67)\end{array}$ & 0.058 \\
\hline \multicolumn{5}{|c|}{ Distant metastasis } \\
\hline $\begin{array}{l}\text { Yes } \\
\text { No }\end{array}$ & $\begin{array}{l}19(26.76) \\
52(73.24)\end{array}$ & $\begin{array}{l}15(78.95) \\
21(40.38)\end{array}$ & $\begin{array}{c}4(21.05) \\
31(59.62)\end{array}$ & $0.004^{*}$ \\
\hline
\end{tabular}

\section{Cell culture and transfection}

The CRC cell lines (LOVO, HCT116, SW480, and SW620) and normal human intestinal epithelial cells (HIEC-6) were obtained from American Type Cell Culture (ATCC, Manassas, USA) and cultured in RPMI-1640 medium (Gibco, Waltham, USA) containing $10 \%$ fetal bovine serum (FBS; Gibco) and $1 \%$ penicillin-streptomycin at $37^{\circ} \mathrm{C}$ with $5 \% \mathrm{CO}_{2}$. Lentiviral short hairpin RNA (shRNA) against FEZF1-AS1 (sh-FEZF1-AS1) and its negative control (sh-NC), miR-363-3p mimic and inhibitor (miR-363-3p and anti-miR-363-3p) or their negative controls (miR-NC and anti-NC), FEZF1AS1 and paired-related homeobox 1 (PRRX1) overexpression vectors or their negative controls (vector) were synthesized by Genechem (Shanghai, China). Cell transfection was performed using Lipofectamine 3000 (Invitrogen, Carlsbad, USA) according to the manufacturer's instructions.

\section{qRT-PCR}

Total RNA was isolated using TRIzol reagent (Invitrogen) and cDNA was synthesized using the Reverse Transcription Kit (HaiGene, Harbin, China). Quantitative real-time PCR (qRT-PCR) was carried out using SYBR Green reagent (HaiGene); $\beta$-actin or U6 was used as the endogenous control. The primers sequences that were used are as follows: FEZF1AS1, Forward (F), 5'-AGGGGATCGACGAGTTGAGA-3' and Reverse (R), 5'-TTGTCCCCGAGTCATTGGTG-3'; 
PRRX1, F, 5'-TGATGCTTTTGTGCGAGAAGA-3' and R, 5'-AGGGAAGCGTTTTTATTGGCT-3'; $\beta$-actin, F, 5'-CATGTACGTTGCTATCCAGGC-3' and R, 5'-CTCCTTAATGTCACGCACGAT-3'; miR-363-3p, F, 5'-TCGGCAGGAATTGCACGGTATCCA-3' and R, 5'-CTCAACTGGTGTCGTGGA-3'; U6 F 5'-GCTTCGGCAGCACATATACTAAAAT-3', R 5'-CGCTTCACGAATTTGCGTGTCAT-3'. Relative expression was determined using the $2^{-\Delta \Delta C t}$ method.

\section{CCK-8 assay}

LOVO and HCT116 cells were seeded into 96-well plates. At a specific time point ( $0 \mathrm{~h}, 24 \mathrm{~h}, 48 \mathrm{~h}$, and $72 \mathrm{~h})$, an equal amount of Cell Counting Kit-8 (CCK-8) solution (Beyotime, Shanghai, China) was added to each well. The proliferation ability of cells was measured at an absorbance of $450 \mathrm{~nm}$.

\section{Transwell assay}

Briefly, 8- $\mu \mathrm{m}$ polycarbonate membranes of Transwell chambers (Corning Inc., Corning, USA) were coated with BD Matrigel (Corning Inc.) for cell invasion, and uncoated for cell migration. Serum-free media was filled in the upper chambers, while media containing 10\% FBS was added to the lower chambers. After $24 \mathrm{~h}$, the cells were fixed and stained with crystal violet. The numbers of invading or migrating cells were counted with an inverted light microscope at $\times 100$ magnification (Shoif, Shanghai, China).

\section{Western blot analysis}

The cells were lysed with lysis buffer (Beyotime). Proteins were separated using sodium dodecyl sulphate-polyacrylamide gel electrophoresis (SDS-PAGE) and transferred onto polyvinylidene fluoride (PVDF) membranes. After blocking with skim milk powder, the membranes were incubated with anti-E-cadherin (1:500; Yubo Bio, Shanghai, China), anti-N-cadherin (1:1000; Yubo), anti-vimentin (1:750; Yubo), anti-PRRX1 (1:500; Yubo), or anti- $\beta$-actin (1:750; Yubo) overnight at $4^{\circ} \mathrm{C}$. Then, membrane were incubated with an horseradish peroxidase (HRP)-conjugated secondary antibody (1:1000; Yubo), and protein signals were observed using electrochemiluminescence ECL solution (Beyotime).

\section{Dual-luciferase reporter assay}

The wild-type (WT) and mutant-type (MUT) reporter vectors of FEZF1-AS1 or PRRX1 were constructed using pmirGLO reporter vector. Lipofectamine 3000 was used to transfect the above vectors into CRC cells with miR363-3p mimic and miR-NC. Luciferase activities were measured using the Dual-Luciferase Assay Kit (Yubo) after transfection for $48 \mathrm{~h}$.

\section{Animal experiments}

HCT116 cells transfected with sh-FEZF1-AS1 or sh-NC were administered subcutaneously into male BALB/c nude mice (purchased from Shanghai Laboratory Animals Center, Shanghai, China). Tumor volumes were calculated every 5 days. After 30 days, the tumors were removed for further analysis. All experiments involving animals were authorized by the Animal Care Committee of the Third Hospital Affiliated to Hebei Medical University and performed according to the Guide for the Care and Use of Laboratory Animals.

\section{Statistical analyses}

All experiments were performed in triplicate. Data are presented as the mean \pm standard deviation (SD) and analyzed using a Student's t-test or one-way analysis of variance (ANOVA) followed by Tukey's post hoc test. Statistical analyses were performed using GraphPad Prism v. 5.0 software (GraphPad Software, San Diego, USA). According to the median cutoff of FEZF1-AS1 expression, patients were divided into low and high groups for analysis. The $X^{2}$ test was used to estimate the correlation between FEZF1-AS1 expression and clinicopathological parameters of CRC patients. Kaplan-Meier analysis was used to analyze the association between FEZF1-AS1 expression and the overall survival of CRC patients, determined by the logrank test. A value of $\mathrm{p}<0.05$ was defined as statistically significant.

\section{Results}

\section{FEZF1-AS1 is highly expressed in CRC}

Compared to normal adjacent tissues, we discovered that FEZF1-AS1 was upregulated in CRC tissues (Fig. 1A). Based on the median FEZF1-AS1 expression in CRC tissues, we divided tissues into those with high FEZF1AS1 expression and low FEZF1-AS1 expression. Analysis of the correlations between FEZF1-AS1 and clinicopathological parameters of CRC patients demonstrated that high FEZF1-AS1 expression was positively correlated with TNM stage, tumor grade and distant metastasis in CRC patients (Table 1). Kaplan-Meier analysis revealed that high FEZF1-AS1 expression was associated with poor overall survival of CRC patients (Fig. 1B). Moreover, we also analyzed the expression of FEZF1-AS1 in CRC cell lines in vitro (LOVO, HCT116, SW480, and SW620) and normal intestinal epithelial cells (HIEC-6), and discovered that FEZF1-AS1 expression was promoted in 4 CRC cell lines (especially in LOVO and HCT116 cells) compared to that in HIEC-6 cells (Fig. 1C). These data suggest that FEZF1-AS1 could act as an oncogene in CRC. 

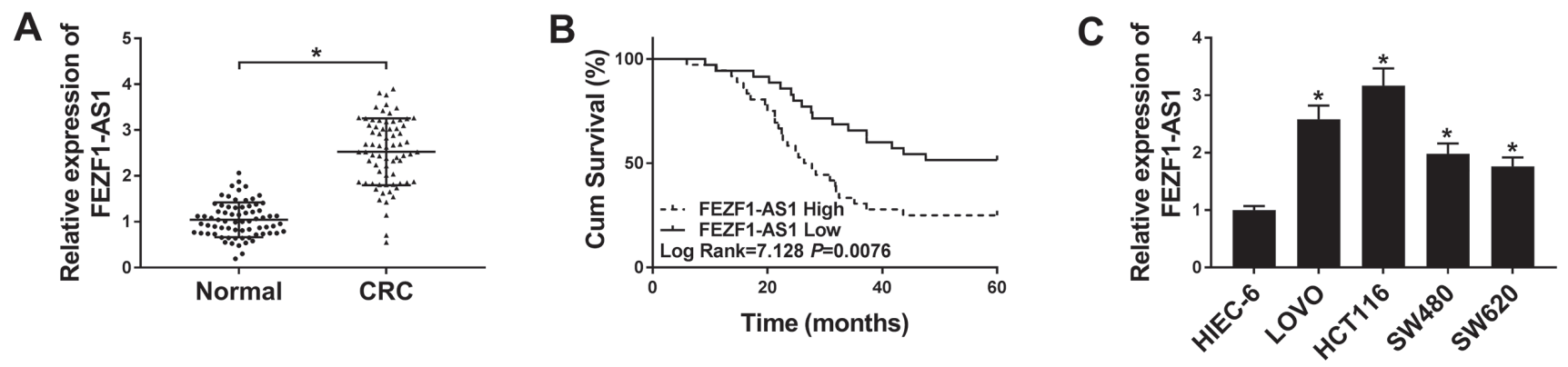

Fig. 1. FEZF1-AS1 expression in colorectal cancer (CRC) tissues and cells. A. Expression of FEZF1-AS1 in CRC tissues (CRC, $n=71)$ and adjacent normal tissues (normal, $n=71$ ) was shown using quantitative real-time polymerase chain reaction (qRT-PCR); B. Kaplan-Meier survival analysis was used to investigate the overall survival of all CRC patients according to FEZF1-AS1 expression; C. FEZF1-AS1 expression in CRC cells (LOVO, HCT116, SW480, and SW620) and normal human intestinal epithelial cells (HIEC-6) was detected using qRT-PCR, * $p<0.05$

A

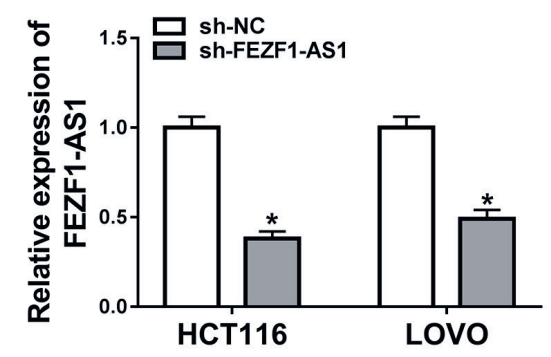

B

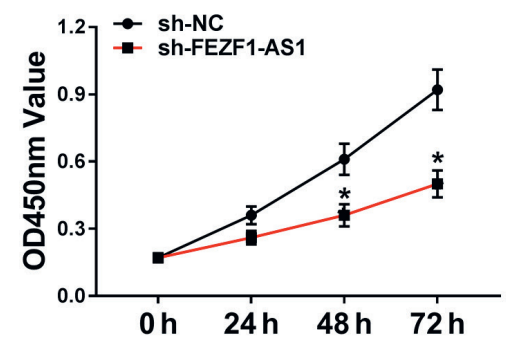

C

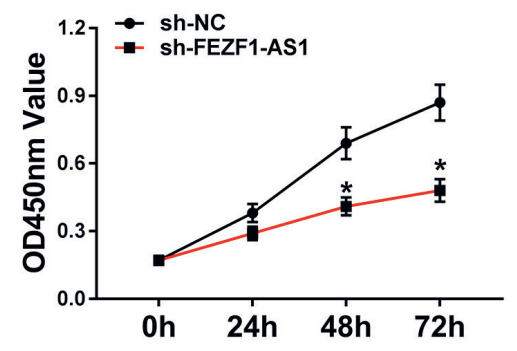

D
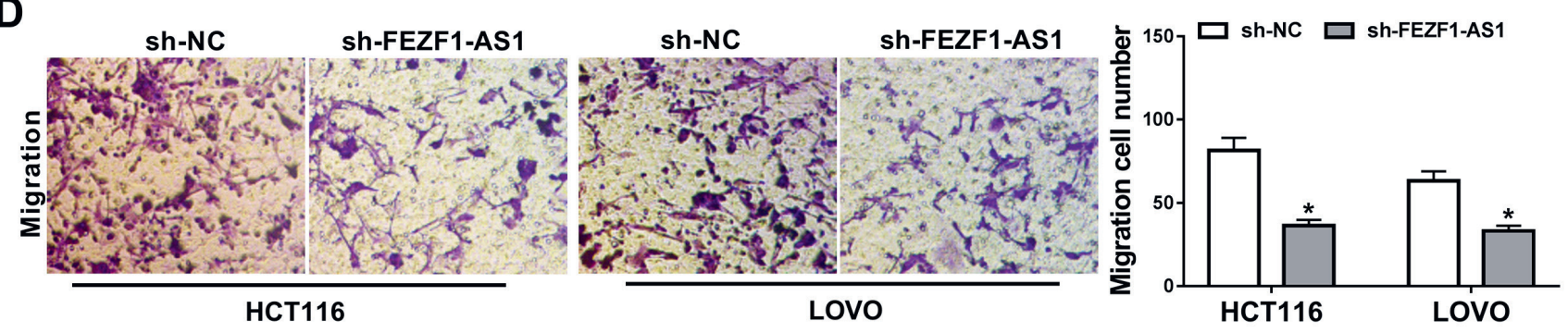

E
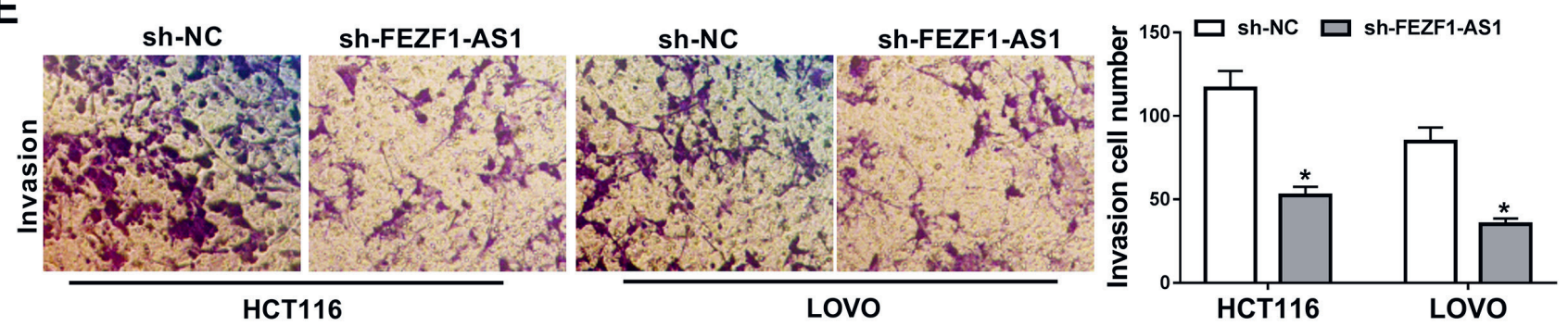

$\mathbf{F}$

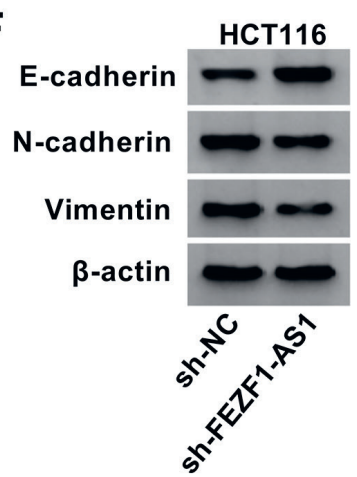

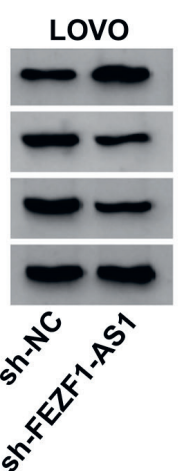
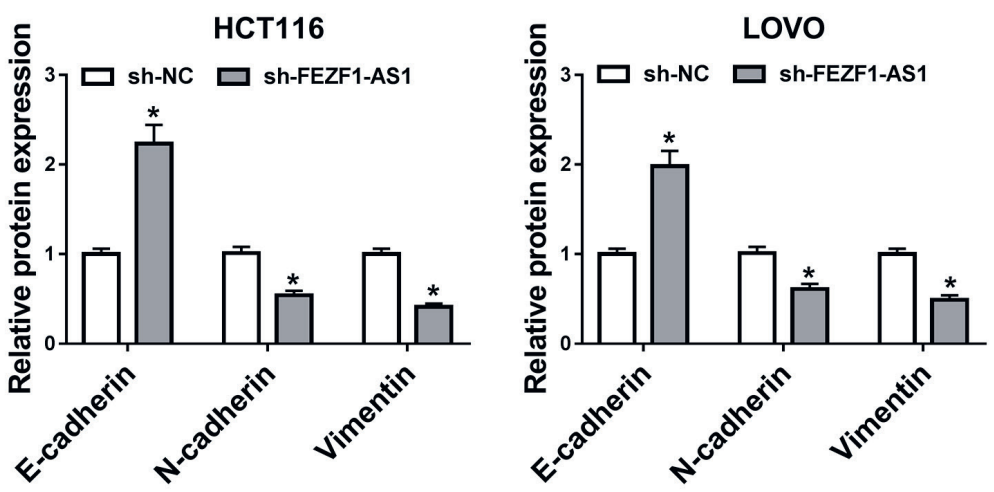

Fig. 2. FEZF1-AS1 knockdown regulated colorectal cancer (CRC) progression. HCT116 and LOVO cells were transfected with sh-FEZF1-AS1 and negative control (sh-NC). A. The quantitative real-time polymerase chain reaction (qRT-PCR) was used to measure FEZF1-AS1 expression; B and C. CRC cell proliferation was measured with Cell Counting Kit-8 (CCK-8) assay; D and E. Representative images and statistical results described cell migration and invasion abilities; F. Western blot analysis was performed to assess E-cadherin, $\mathrm{N}$-cadherin and vimentin protein level, ${ }^{*} \mathrm{p}<0.05$ 
A

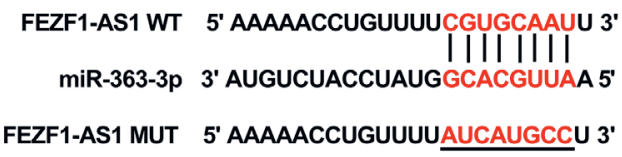

FEZF1-AS1 MUT 5' AAAAACCUGUUUUAUCAUGCCU 3'

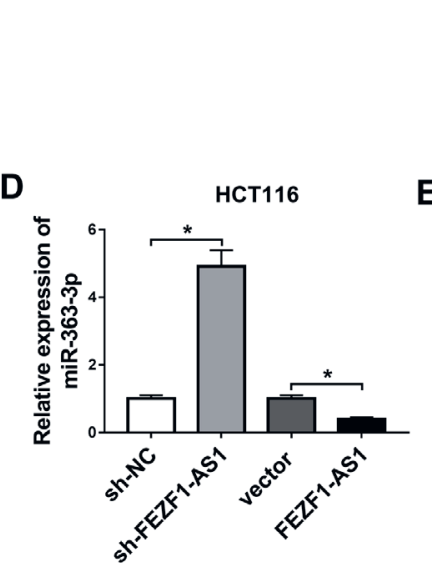

\section{B}
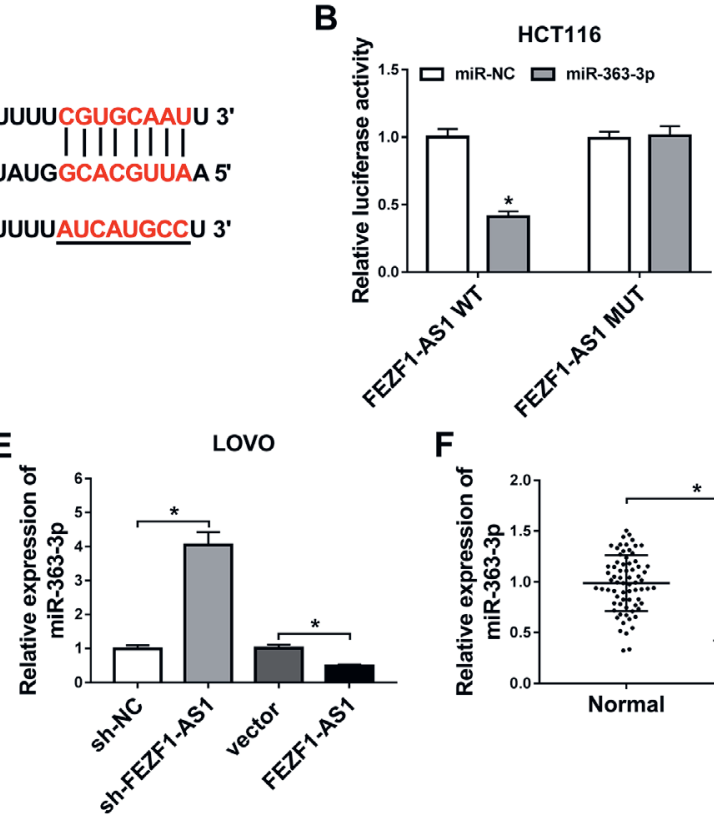

$\mathbf{F}$

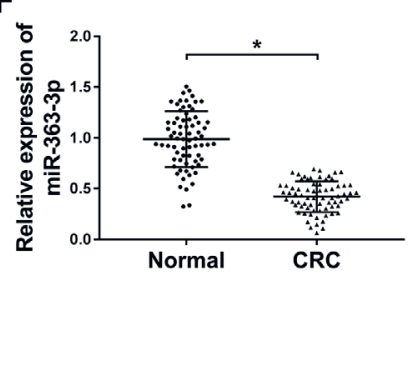

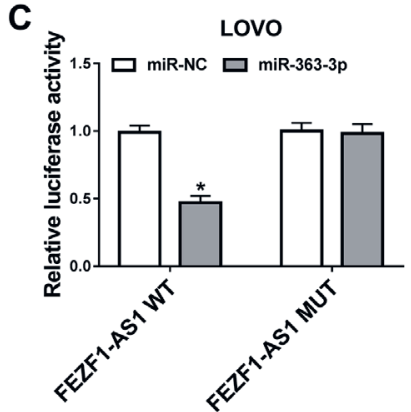

G

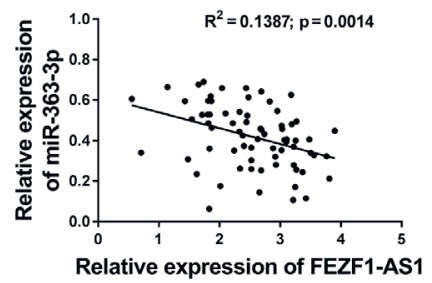

Fig. 3. MiR-363-3p could interact with FEZF1-AS1. A. The binding sites between FEZF1-AS1 and miR-363-3p; B and C. The interaction between FEZF1-AS1 and miR-363-3p was confirmed using dual-luciferase reporter assay; D and E. The expression of miR-363-3p in HCT116 and LOVO cells transfected with sh-FEZF1AS1 and FEZF1-AS1 overexpression vector was determined using quantitative real-time polymerase chain reaction (qRT-PCR); F. miR-363-3p expression in colorectal cancer $(C R C)$ tissues $(C R C, n=71$ ) and adjacent normal tissues (normal, $n=71$ ) was detected using qRT-PCR; $G$. The correlation analysis between miR-363-3p and FEZF1-AS1 expression were determined with Pearson correlation coefficient analysis, ${ }^{*} p<0.05$

\section{FEZF1-AS1 knockdown hindered CRC proliferation, migration, invasion, and EMT}

To explore the effects of IncRNA FEZF1-AS1 on CRC progression, FEZF1-AS1 expression was silenced in HCT116 and LOVO cells using sh-FEZF1-AS1. The quantitative real-time polymerase chain reaction (qRT-PCR) results indicated that sh-FEZF1-AS1 had a significant silencing capacity on FEZF1-AS1 expression in both cells (Fig. 2A). The CCK- 8 assay demonstrated that compared with the sh-NC group, downregulation of FEZF1-AS1 could suppress cell proliferation in HCT116 and LOVO cells (Fig. 2B,C). Furthermore, the ability of cells to migrate and invade was significantly reduced following silencing FEZF1-AS1 in HCT116 and LOVO cells (Fig. 2D,E). At the same time, we explored the levels of EMT-related proteins and found that the epithelial marker E-cadherin level was increased, while mesenchymal markers $\mathrm{N}$-cadherin and vimentin levels were decreased in HCT116 and LOVO cells following FEZF1-AS1 silencing (Fig. 2F). Hence, these data demonstrated that silenced FEZF1-AS1 could hinder the proliferation, metastasis and EMT of CRC.

\section{FEZF1-AS1 acted as miR-363-3p sponge}

To further study the mechanism of FEZF1-AS1 in CRC progression, we predicted the potential targeted miRNAs of FEZF1-AS1 using the StarBase tool (http://starbase.sysu. edu.cn/). As shown in Fig. 3A, the potential complementary binding sites between FEZF1-AS1 and miR-363-3p are presented, and the WT and MUT FEZF1-AS1 vectors were constructed. Dual-luciferase reporter assay results showed that miR-363-3p mimic restrained the luciferase activity of FEZF1-AS1 WT vector, while not affecting that of the FEZF1-AS1 MUT vector in HCT116 and LOVO cells (Fig. 3B,C). Furthermore, we explored the impact of FEZF1-AS1 expression on miR-363-3p, and showed that FEZF1-AS1 knockdown could promote, while overexpression could inhibit the expression of miR-363-3p (Fig. 3D,E). Furthermore, we determined that miR-363-3p had a lower expression in CRC tissues (Fig. 3F), and correlation analysis showed that this was negatively correlated to FEZF1-AS1 expression (Fig. 3G). These results revealed that miR-363-3p could interact with FEZF1-AS1 in CRC cells.

\section{MiR-363-3p inhibition reversed the suppressive effect of FEZF1-AS1 silencing on CRC progression}

Subsequently, we verified the effect of miR-363-3p expression on CRC progression and confirmed whether FEZF1-AS1 regulated CRC progression by sponging miR-363-3p. Here, miR-NC or miR-363-3p mimic were transfected into HCT116 and LOVO cells, and sh-FEZF1-AS1 and anti-miR-363-3p were co-transfected into the same cells. MiR-363-3p mimic markedly increased miR-363-3p expression, and its inhibitor could also significantly reduce miR-363-3p expression in the presence of sh-FEZF1-AS1 (Fig. 4A,B). Results of the CCK- 8 assay indicated that overexpression of miR363-3p blocked the proliferation of CRC cells, while 

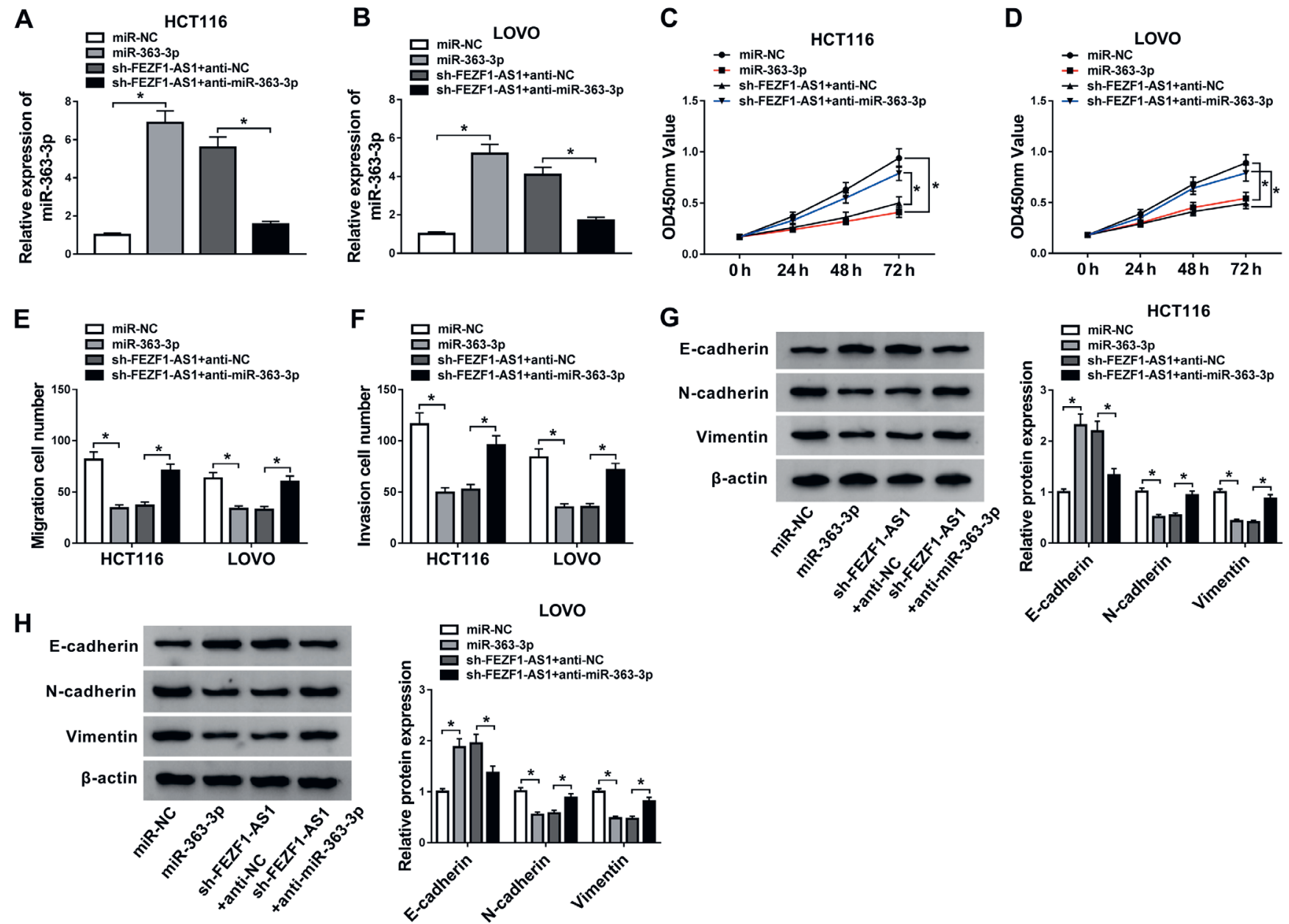

Fig. 4. miR-363-3p and FEZF1-AS1 regulated the progression of colorectal cancer (CRC) cells. The miR-363-3p mimic, miR-NC, sh-FEZF1-AS1, anti-NC, or antimiR-363-3p were transfected into HCT116 and LOVO cells. A and B. Expression of miR-363-3p was determined using quantitative real-time polymerase chain reaction ( $\mathrm{QRT} T-\mathrm{PCR}$ ); C and D. Cell proliferation following transfection was measured with Cell Counting Kit-8 (CCK-8) assay; E and F. Cell migration and invasion of transfected cells in transwell assays; $\mathrm{G}$ and $\mathrm{H}$. Protein levels of E-cadherin, $\mathrm{N}$-cadherin and vimentin as assessed with western blot analysis, ${ }^{*} \mathrm{p}<0.05$

anti-miR-363-3p could inhibit CRC cell proliferation, as promoted by FEZF1-AS1 silencing (Fig. 4C,D). Similarly, transwell assay also revealed that miR-363-3p overexpression could hinder HCT116 and LOVO cell migration and invasion, while its inhibitor also could abolish the suppression effect of FEZF1-AS1 knockdown (Fig. 4E,F). Moreover, western blot analysis showed that the miR-363-3p increased E-cadherin protein level, while decreasing $\mathrm{N}$-cadherin and vimentin protein levels in HCT116 and LOVO cells. Finally, miR-363-3p inhibitor could reverse the increased E-cadherin expression and the decreasing effect on $\mathrm{N}$-cadherin and vimentin expression when FEZF1-AS1 was knocked down (Fig. 4G,H). Hence, these data indicated that FEZF1AS1 sponged miR-363-3p to regulate CRC progression.

\section{PRRX1 was a target of miR-363-3p}

Then, StarBase tool was used to predict the downstream target of miR-363-3p, and PRRX13'UTR was found to have binding sites for miR-363-3p (Fig. 5A). Further experiments identified that miR-363-3p mimic suppressed the luciferase activity of PRRX1 WT vector in HCT116 and LOVO cells, while not affecting that of the PRRX1 MUT vector (Fig. 5B,C). Furthermore, western blot analysis demonstrated that the protein level of PRRX1 was significantly reduced by miR-363-3p overexpression, and promoted by miR-363-3p inhibition in HCT116 and LOVO cells (Fig. 5D,E). These data suggested that miR-363-3p could target PRRX1 in CRC cells.

\section{PRRX1 overexpression reversed the regulation of sh-FEZF1-AS1 on CRC progression}

To verify whether PRRX1 was involved in the regulation of FEZF1-AS1 on CRC cell progression, sh-FEZF1-AS1 and PRRX1 overexpression vector were co-transfected into HCT116 and LOVO cells. The detection of PRRX1 protein level showed that knockdown of FEZF1-AS1 decreased the level of PRRX1, while this effect could be reversed by the overexpression of PRRX1 (Fig. 6A,B). The CCK- 8 and transwell assays revealed that the aberrant overexpression of PRRX1 recovered the inhibiting 
A

PRRX1 WT 3'UTR 5' CCACCCCUCACCCUAUGCAAUC 3' IIIIII

miR-363-3p 3' AUGUCUACCUAUGGCACGUUAA 5'

PRRX1 MUT 3'UTR 5' CCACCCCUCACCCUAGAUCGCC 3'
B

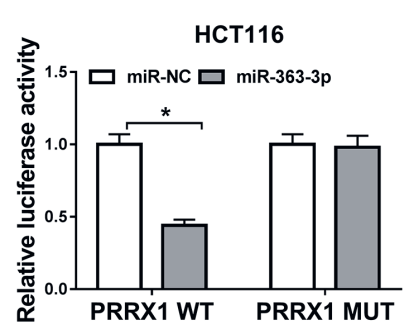

C

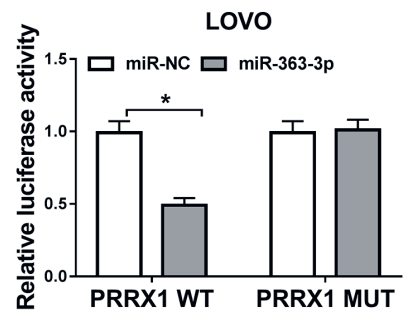

D

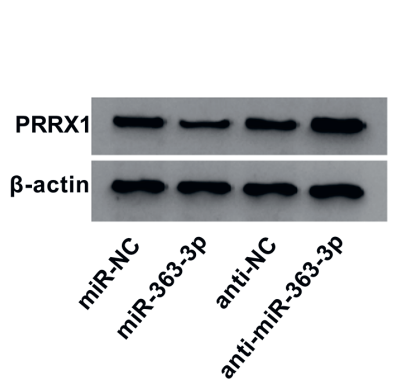

HCT116

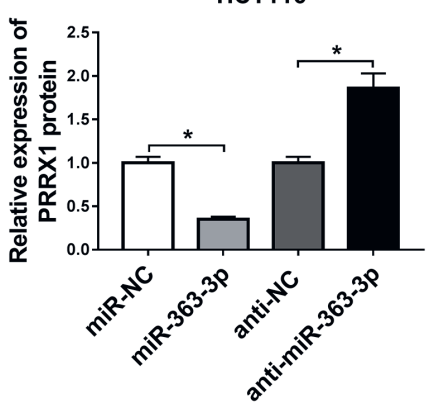

E

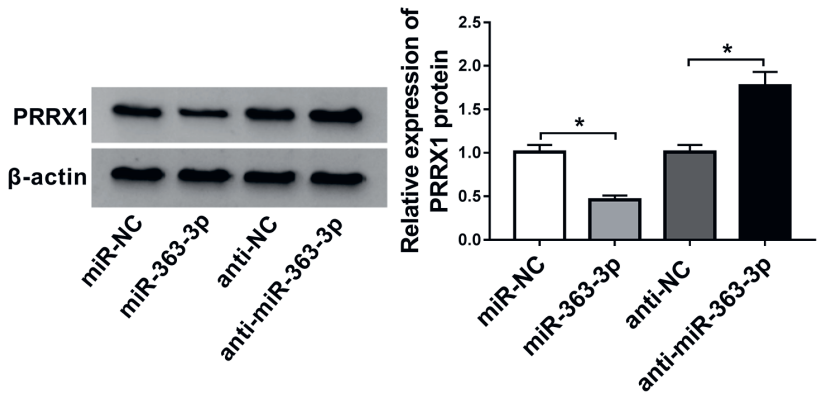

Fig. 5. PRRX1 was a target of miR-363-3p; A. The sequences of PRRX1-WT and PRRX1-MUT; B and C. Dual-luciferase reporter assay was employed to assess the interaction between PRRX1 and miR-363-3p; D and E. The protein level of PRRX1 in HCT116 and LOVO cells transfected with miR-363-3p mimic or inhibitor were examined with western blot analysis, ${ }^{*} p<0.05$

A

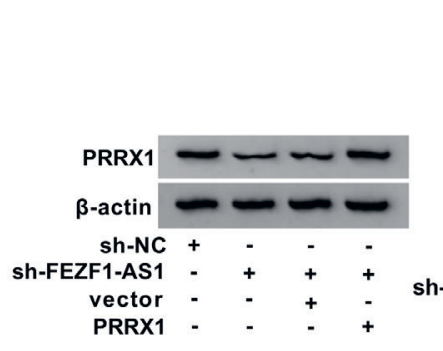

C
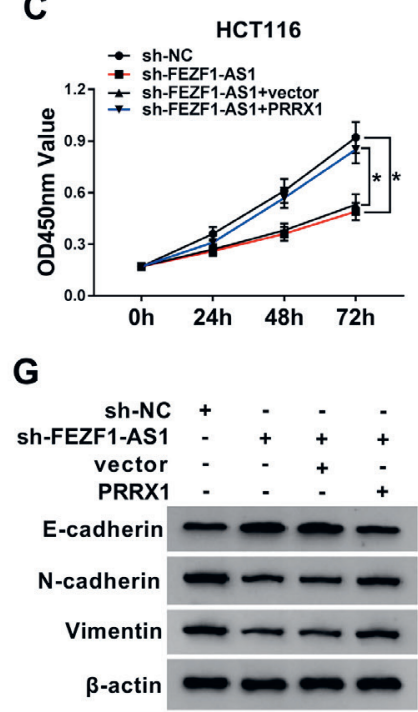

HCT116

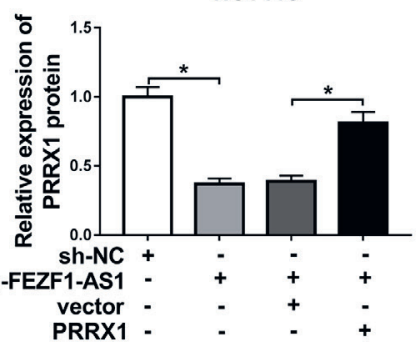

D
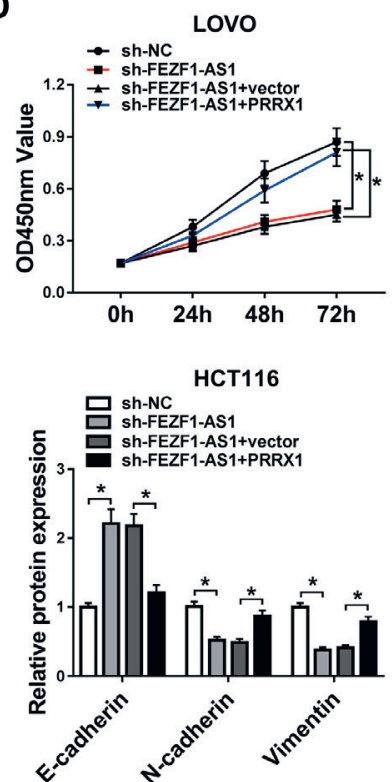

B

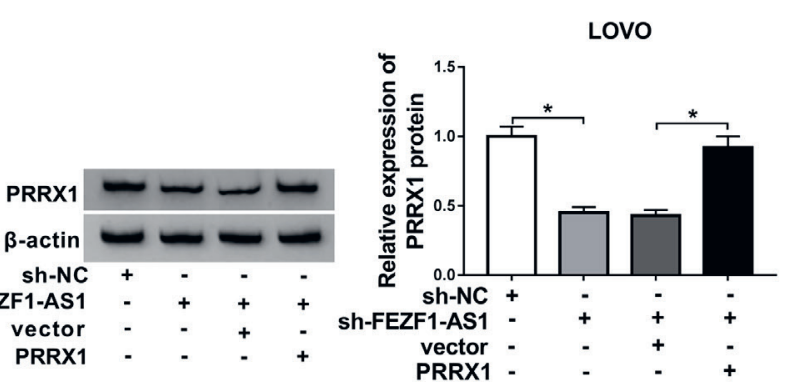

E

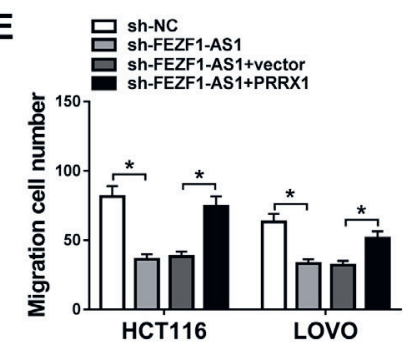

F 吕 $\begin{aligned} & \text { Sh-NC } \\ & \text { sh-FEF1-AS1 }\end{aligned}$

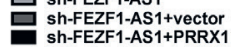

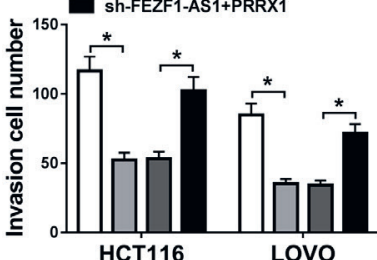

H

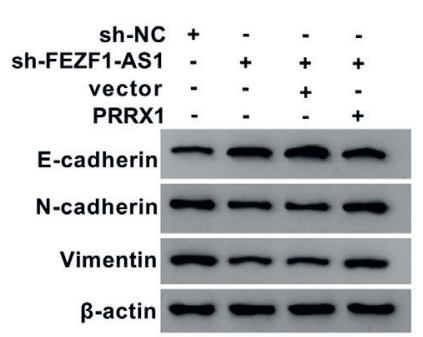

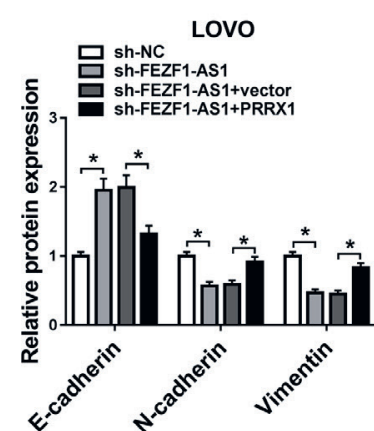

Fig. 6. PRRX1 overexpression regulated colorectal cancer (CRC) progression. HCT116 and LOVO cells were transfected with negative control (sh-NC), sh-FEZF1-AS1, sh-FEZF1-AS1 + vector, or sh-FEZF1-AS1 + PRRX1. A and B. Protein levels of PRRX1 as detected with western blot analysis; $C$ and D. Cell proliferation of transfected cells using a Cell Counting Kit-8 (CCK-8) assay; E and F. Cell migration and invasion of transfected cells as measured using transwell assay; G and H. Western blot analysis was used to determine the E-cadherin, $\mathrm{N}$-cadherin and vimentin protein levels, ${ }^{*} \mathrm{p}<0.05$ 
A

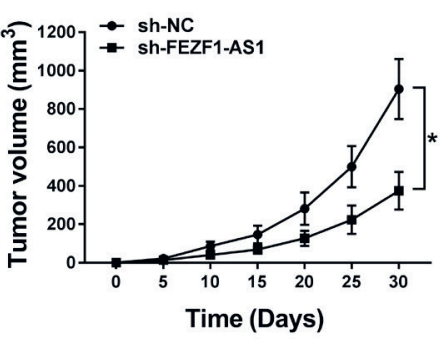

B

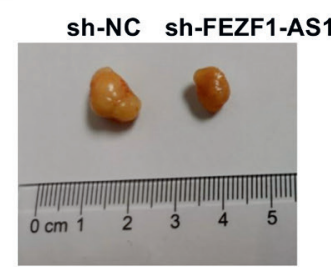

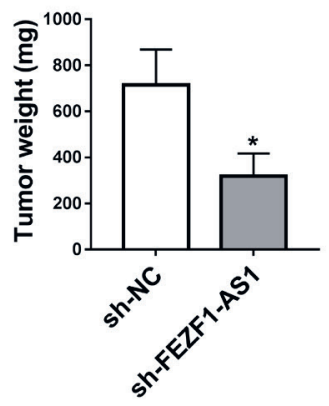

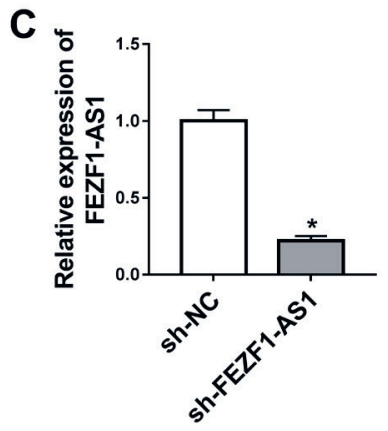

D

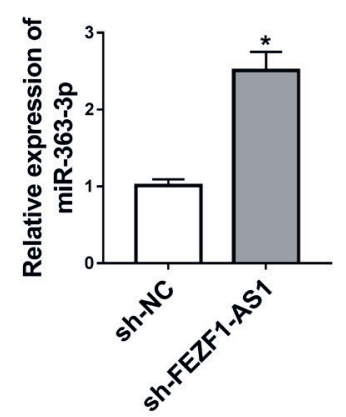

E PRRX1

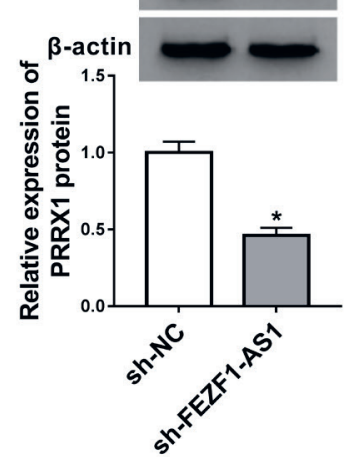

$\mathbf{F}$

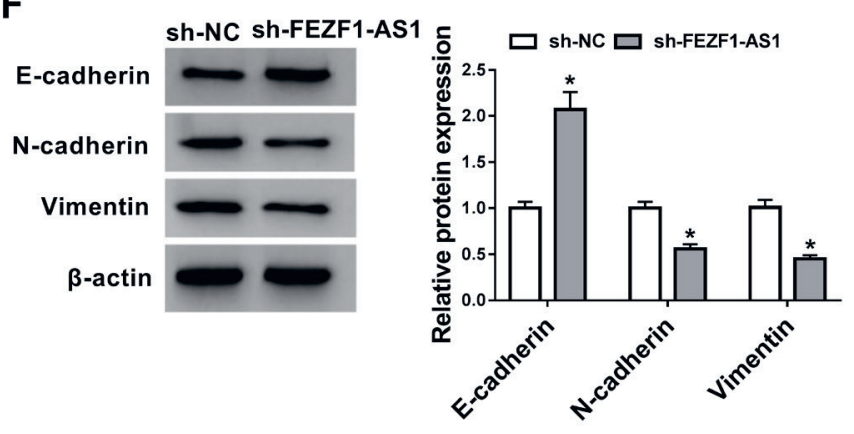

Fig. 7. FEZF1-AS1 silencing regulated colorectal cancer (CRC) tumor growth. Tumor volume (A) and tumor weight (B) were measured in mouse xenograft models; C and D. The FEZF1-AS1 and miR-363-3p expression levels were examined using quantitative real-time polymerase chain reaction (qRT-PCR); $\mathrm{E}, \mathrm{F}$. The PRRX1, E-cadherin, $\mathrm{N}$-cadherin, and vimentin protein levels were measured using western blot analysis, ${ }^{*} \mathrm{p}<0.05$

effects of FEZF1-AS1 knockdown on CRC cell proliferation, migration and invasion (Fig. 6C-F). Furthermore, PRRX1 overexpression also reversed the promoting effect of FEZF1-AS1 knockdown on E-cadherin expression, and the inhibiting effect on $\mathrm{N}$-cadherin and vimentin expression in HCT116 and LOVO cells (Fig. 6G,H). The above results suggest that PRRX1 participated in the regulation of FEZF1-AS1 on CRC progression.

\section{Interference of FEZF1-AS1 reduced CRC tumor growth}

To further confirm the influence of FEZF1-AS1 on CRC progression, HCT116 cells transfected with sh-FEZF1-AS1 were subcutaneously injected into nude mice. Following 30 days of monitoring, the tumor volume of the sh-FEZF1AS1 group was reduced when compared to the sh-NC group (Fig. 7A). Furthermore, tumor weight also reduced in the sh-FEZF1-AS1 group (Fig. 7B). Through detecting the expression of FEZF1-AS1 and miR-363-3p in the tumors, we found that FEZF1-AS1 expression was significantly decreased (Fig. 7C), while miR-363-3p was markedly increased in the tumor tissues of the sh-FEZF1-AS1 group (Fig. 7D). Moreover, western blot analysis showed that FEZF1-AS1 knockdown could block the protein level of PRRX1 in tumor tissues of mice (Fig. 7E), promote the Ecadherin expression and reduce the $\mathrm{N}$-cadherin and vimentin expression (Fig. 7F). These results confirmed that FEZF1-AS1 had a cancer-promoting effect in CRC.

\section{Discussion}

Recent studies have shown that IncRNAs functioned as tumor suppressors or promoters in CRC development and progression. For example, IncRNA MIR17HG promoted CRC progression, ${ }^{19}$ and IncRNA ROR1-AS1 enhanced CRC cell metastasis and proliferation, both of which played a role as oncogenes in CRC. ${ }^{20}$ However, lncRNA GAS5 inhibited metastasis and promoted autophagy of CRC cells to suppress CRC. ${ }^{21}$ In other CRC-related investigations, Bian et al. found that FEZF1-AS1 could bind to the PKM2 protein to promote aerobic glycolysis and activate STAT3 signaling to accelerate the progression of CRC. ${ }^{22}$ Also, Chen et al. proposed that FEZF1-AS1 may promote FEZF1 and in turn facilitate CRC proliferation, migration and invasion. ${ }^{23} \mathrm{Simi}-$ lar to these findings, our data confirmed that levels of $\ln$ cRNA FEZF1-AS1 were significantly upregulated in CRC. Higher FEZF1-AS1 was related to TNM stage, tumor grade, distant metastasis, and overall survival rate of CRC patients. Functional experiments showed that FEZF1-AS1 knockdown restrained CRC cell proliferation, metastasis and EMT, and decreased CRC tumor growth. Consistent with the results of previous studies, ${ }^{22,23}$ our results verified that FEZF1-AS1 could be used as an oncogene in CRC, similar to its role in other cancers. ${ }^{24,25}$

To investigate the function of FEZF1-AS1 as a ceRNA, we performed bioinformatics predictions and found that miR-363-3p had complementary binding sites. Previous research has indicated that miR-363-3p took part 
in the regulation of various cancers, including glioma, osteosarcoma and gastric cancer. ${ }^{26-28}$ Furthermore, miR-363-3p has been shown to be related to the EMT process. ${ }^{29,30}$ In this, miR-363-3p was confirmed to have decreased expression in CRC. FEZF1-AS1 silencing hindered miR-363-3p level in vitro and in vivo. Moreover, knockdown of FEZF1-AS1 had the same effect as miR363-3p overexpression, both of which could promote the level of miR-363-3p and block proliferation, metastasis and EMT of CRC cells, while the miR-363-3p inhibitor could reverse the these effects. Therefore, we confirmed that miR-363-3p, as the absorption target of FEZF1-AS1, participates in the regulation of CRC progression though interactions with FEZF1-AS1.

Paired-related homeobox 1 was found to be an EMT inducer that participates in the metastasis of various cancers. ${ }^{31,32}$ Investigations by Takahashi et al. suggested that PRRX1 induced EMT and was involved in metastasis and poor prognosis of CRC patients. ${ }^{33}$ Interestingly, through prediction and verification, we discovered that PRRX1 was a target of miR-363-3p. Furthermore, we also found that PRRX1 overexpression reversed the inhibitory functions of FEZF1-AS1 silencing on CRC cell proliferation, metastasis and EMT. Additionally, we discovered that PRRX1 expression was positively regulated by FEZF1-AS1 in vitro and in vivo. These data suggested that FEZF1-AS1 regulated CRC progression by mediating the miR-363-3p/ PRRX1 axis.

\section{Limitations}

The limitation of this study is that we did not further detect classical signaling pathways that might be involved in the modulation of FEZF1-AS1/miR-363-3p/PRRX1 axis in CRC.

\section{Conclusions}

Our studies suggested that lncRNA FEZF1-AS1 upregulated PRRX1 by sponging miR-363-3p to promote the proliferation, metastasis and EMT of CRC. The discovery of the FEZF1-AS1/miR-363-3p/PRRX1 axis elucidated the mechanism of FEZF1-AS1 in CRC and provided new avenues of investigation into the determination of CRC therapeutic targets.

\section{ORCID iDs}

Tongtong Zhang (1) https://orcid.org/0000-0002-1121-021X Suyang Yu (i) https://orcid.org/0000-0002-9034-9294 Shipeng Zhao (1) https://orcid.org/0000-0003-4522-2208

\section{References}

1. Brenner H, Kloor M, Pox CP. Colorectal cancer. Lancet. 2014;383(9927): 1490-1502. doi:10.1016/S0140-6736(13)61649-9

2. Siegel RL, Miller KD, Fedewa SA, et al. Colorectal cancer statistics, 2017. CA Cancer J Clin. 2017;67(3):177-193. doi:10.3322/caac.21395
3. Torre LA, Bray F, Siegel RL, Ferlay J, Lortet-Tieulent J, Jemal A. Global cancer statistics, 2012. CA Cancer J Clin. 2015;65(2):87-108. doi:10. 3322/caac. 21262

4. Qi L, Ding Y. Analysis of metastasis associated signal regulatory network in colorectal cancer. Biochem Biophys Res Commun. 2018;501(1): 113-118. doi:10.1016/j.bbrc.2018.04.186

5. Huang R, Zong X. Aberrant cancer metabolism in epithelial-mesenchymal transition and cancer metastasis: Mechanisms in cancer progression. Crit Rev Oncol Hematol. 2017;115:13-22. doi:10.1016/j.critrevonc. 2017.04.005

6. Bhan A, Soleimani M, Mandal SS. Long noncoding RNA and cancer: A new paradigm. Cancer Res. 2017;77(15):3965-3981. doi:10.1158/00085472.CAN-16-2634

7. Wei L, Wang X, Lv L, Zheng Y, Zhang N, Yang M. The emerging role of noncoding RNAs in colorectal cancer chemoresistance. Cell Oncol. 2019;42(6):757-768. doi:10.1007/s13402-019-00466-8

8. Boon RA, Jae N, Holdt L, Dimmeler S. Long noncoding RNAs: From clinical genetics to therapeutic targets? J Am Coll Cardiol. 2016;67(10): 1214-1226. doi:10.1016/j.jacc.2015.12.051

9. Tay Y, Rinn J, Pandolfi PP. The multilayered complexity of ceRNA crosstalk and competition. Nature. 2014;505(7483):344-352. doi:10.1038/ nature12986

10. Wilusz JE, Sunwoo H, Spector DL. Long noncoding RNAs: Functional surprises from the RNA world. Genes Dev. 2009;23(13):1494-1504. doi:10.1101/gad.1800909

11. Jiang $P$, Han X, Zheng Y, Sui J, Bi W. Long non-coding RNA NKILA serves as a biomarker in the early diagnosis and prognosis of patients with colorectal cancer. Oncol Lett. 2019;18(2):2109-2117. doi:10.3892/ ol.2019.10524

12. Ren $\mathrm{Y}, \mathrm{Zhao} \mathrm{C}, \mathrm{He} \mathrm{Y}, \mathrm{Xu} \mathrm{H}$, Min X. Long non-coding RNA bladder cancer-associated transcript 2 contributes to disease progression, chemoresistance and poor survival of patients with colorectal cancer. Oncol Lett. 2019;18(2):2050-2058. doi:10.3892/ol.2019.10487

13. Wang W, Xie Y, Chen F, et al. LncRNA MEG3 acts a biomarker and regulates cell functions by targeting ADAR1 in colorectal cancer. World J Gastroenterol. 2019;25(29):3972-3984. doi:10.3748/wjg.v25.i29.3972

14. Xu L, Hou TJ, Yang P. Mechanism of IncRNA FEZF1-AS1 in promoting the occurrence and development of oral squamous cell carcinoma through targeting miR-196a. Eur Rev Med Pharmacol Sci. 2019;23(15): 6505-6515. doi:10.26355/eurrev_201908_18534

15. Quan LJ, Wang WJ. FEZF1-AS1 functions as an oncogenic IncRNA in retinoblastoma. Biosci Rep. 2019;39(5):BSR20190754. doi:10.1042/ BSR20190754

16. Zhu LF, Song LD, Xu Q, Zhan JF. Highly expressed long non-coding RNA FEZF1-AS1 promotes cells proliferation and metastasis through Notch signaling in prostate cancer. Eur Rev Med Pharmacol Sci. 2019;23(12):5122-5132. doi:10.26355/eurrev_201906_18176

17. Cheng Y. FEZF1-AS1 is a key regulator of cell cycle, epithelial-mesenchymal transition and Wnt/beta-catenin signaling in nasopharyngeal carcinoma cells. Biosci Rep. 2019;39(1):BSR20180906. doi:10.1042/ BSR20180906

18. Wang YD, Sun XJ, Yin JJ, et al. Long non-coding RNA FEZF1-AS1 promotes cell invasion and epithelial-mesenchymal transition through JAK2/STAT3 signaling pathway in human hepatocellular carcinoma. Biomed Pharmacother. 2018;106:134-141. doi:10.1016/j.biopha.2018. 05.116

19. Xu J, Meng Q, Li X, et al. Long non-coding RNA MIR17HG promotes colorectal cancer progression via miR-17-5p. Cancer Res. 2019;79(19): 4882-4895. doi:10.1158/0008-5472.CAN-18-3880

20. Liao T, Maierdan SL, Lv C. ROR1-AS1 promotes tumorigenesis of colorectal cancer via targeting Wnt/beta-catenin. Eur Rev Med Pharmacol Sci. 2019;23(3 Suppl):217-223. doi:10.26355/eurrev_201908_18650

21. Liu L, Wang HJ, Meng T, et al. IncRNA GAS5 inhibits cell migration and invasion and promotes autophagy by targeting miR-222-3p via the GAS5/PTEN-signaling pathway in CRC. Mol Ther Nucleic Acids. 2019;17:644-656. doi:10.1016/j.omtn.2019.06.009

22. Bian Z, Zhang J, Li M, et al. LncRNA-FEZF1-AS1 promotes tumor proliferation and metastasis in colorectal cancer by regulating PKM2 signaling. Clin Cancer Res. 2018;24(19):4808-4819. doi:10.1158/10780432.CCR-17-2967

23. Chen N, Guo D, Xu Q, et al. Long non-coding RNA FEZF1-AS1 facilitates cell proliferation and migration in colorectal carcinoma. Oncotarget. 2016;7(10):11271-11283. doi:10.18632/oncotarget.7168 
24. Shi C, Sun L, Song Y. FEZF1-AS1: A novel vital oncogenic IncRNA in multiple human malignancies. Biosci Rep. 2019;39(6):BSR20191202. doi:10.1042/BSR20191202

25. Ou ZL, Zhang M, Ji LD, et al. Long noncoding RNA FEZF1-AS1 predicts poor prognosis and modulates pancreatic cancer cell proliferation and invasion through miR-142/HIF-1alpha and miR-133a/EGFR upon hypoxia/normoxia .J Cell Physiol. 2019. doi:10.1002/jcp.28188

26. Xu DX, Guo JJ, Zhu GY, Wu HJ, Zhang QS, Cui T. MiR-363-3p modulates cell growth and invasion in glioma by directly targeting pyruvate dehydrogenase B. Eur Rev Med Pharmacol Sci. 2018;22(16):5230-5239. doi:10.26355/eurrev_201808_15721

27. Wang K, Yan L, Lu F. miR-363-3p Inhibits osteosarcoma cell proliferation and invasion via targeting SOX4. Oncol Res. 2019;27(2):157-163. doi:10.3727/096504018X15190861873459

28. Song B, Yan J, Liu C, Zhou H, Zheng Y. Tumor suppressor role of miR363-3p in gastric cancer. Med Sci Monit. 2015;21:4074-4080. doi:10. 12659/msm.896556
29. Hu F, Min J, Cao X, et al. MiR-363-3p inhibits the epithelial-to-mesenchymal transition and suppresses metastasis in colorectal cancer by targeting Sox4. Biochem Biophys Res Commun. 2016;474(1):35-42. doi:10.1016/j.bbrc.2016.04.055

30. Chang J, Gao F, Chu H, Lou L, Wang H, Chen Y. miR-363-3p inhibits migration, invasion, and epithelial-mesenchymal transition by targeting NEDD9 and SOX4 in non-small-cell lung cancer. J Cell Physiol. 2019;235(2):1808-1820. doi:10.1002/jcp.29099

31. Guo J, Fu Z, Wei J, Lu W, Feng J, Zhang S. PRRX1 promotes epithelial-mesenchymal transition through the Wnt/beta-catenin pathway in gastric cancer. Med Oncol. 2015;32(1):393. doi:10.1007/s12032-0140393-x

32. Hirata H, Sugimachi K, Takahashi Y, et al. Downregulation of PRRX1 confers cancer stem cell-like properties and predicts poor prognosis in hepatocellular carcinoma. Ann Surg Oncol. 2015;22(Suppl 3):S1402S1409. doi:10.1245/s10434-014-4242-0

33. Takahashi Y, Sawada G, Kurashige J, et al. Paired related homoeobox 1, a new EMT inducer, is involved in metastasis and poor prognosis in colorectal cancer. Br J Cancer. 2013;109(2):307-311. doi:10.1038/ bjc.2013.339 